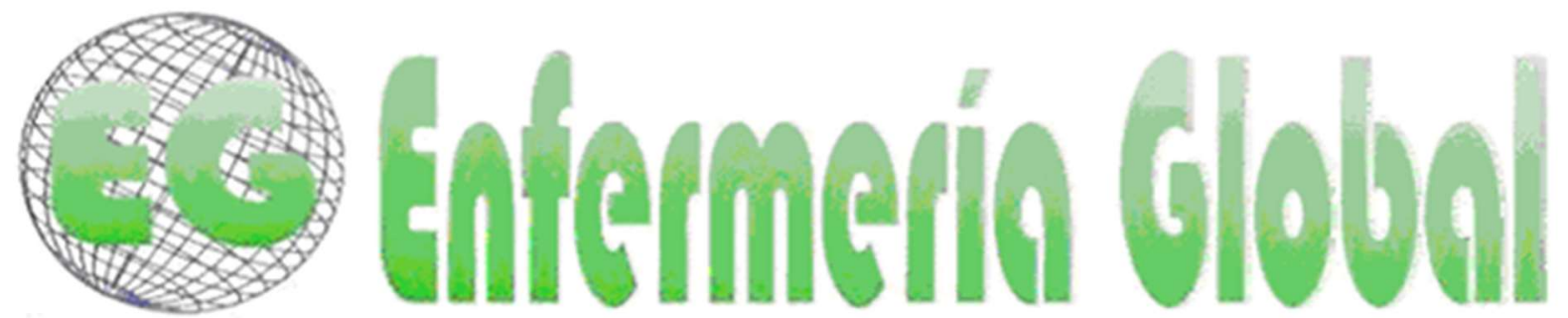

\title{
ORIGINALES
}

\section{Comunicación terapéutica del enfermero, desde la perspectiva del hospitalizado en el hospital General Jaén, Perú}

Therapeutic communication of the nurse, from the vision of the hospitalized in the General hospital Jaén, Peru

María Saavedra Chinchayán ${ }^{1}$

Gladys León Montoya ${ }^{2}$

Adalia Dávila Guerrero ${ }^{3}$

1 Licenciada en Enfermería, Docente en Enfermería. Universidad Nacional Toribio Rodríguez de Mendoza de Amazonas. Chachapoyas - Amazonas, Perú. mariaestsaavedra20@gmail.com

2. Doctora en Salud Pública, Licenciada en Enfermería. Docente investigadora Universidad Nacional Toribio Rodríguez de Mendoza de Amazonas. Chachapoyas - Amazonas, Perú.

3. Docente Universidad Nacional Toribio Rodríguez de Mendoza de Amazonas. Chachapoyas Amazonas, Perú.

\section{https://doi.org/10.6018/eglobal.417451}

Recibido: 5/03/2020

Aceptado: 20/10/2020

\section{RESUMEN:}

Objetivo: Determinar el nivel de comunicación terapéutica del profesional de enfermería desde la perspectiva de las personas hospitalizadas en el servicio de medicina del Hospital General Jaén, Perú.

Material y método: Estudio cuantitativo, nivel descriptivo; observacional, prospectivo, transversal, análisis estadístico univariado, muestra constituida por 122 personas hospitalizadas, se utilizó como instrumento el "Cuestionario de la comunicación terapéutica" elaborado por Lourdes Guevara Chuquillanqui, con validez por juicio de expertos fue $\mathrm{VC}=2.2>\mathrm{VT}=1.6449$ y una confiabilidad de 0.92 .

Resultados: El 100\% (122) de las personas hospitalizadas; $71.3 \%$ (87) percibió una comunicación terapéutica de nivel bajo y el $28.7 \%$ (35) nivel medio. Según las dimensiones: en empatía el $70.5 \%$ (86) percibió una comunicación terapéutica de nivel bajo, el $27 \%$ (33) nivel medio y $2.5 \%$ (3) nivel alto, en la dimensión respeto el $54.9 \%$ (67) percibió una comunicación terapéutica un nivel bajo, el $42.6 \%$ (52) un nivel medio y el $2.5 \%$ (3) nivel alto, en la dimensión escucha receptiva el 54.9\% (67) percibió una comunicación terapéutica de nivel bajo y el $45.1 \%$ (55) nivel medio, en la dimensión emociones del paciente el $57.4 \%$ (70) percibió una comunicación terapéutica de nivel bajo, el 34.4\% (42) nivel medio y el $8.2 \%$ (10) nivel alto, en la dimensión acompañamiento en su reflexión el $68 \%$ (83) percibió una comunicación terapéutica de nivel bajo, el $68 \%$ (83) nivel bajo, el $25.4 \%$ (31) nivel medio y $6.6 \%$ (8) nivel alto.

Conclusiones: La percepción de comunicación terapéutica de las personas hospitalizadas fue de nivel bajo a medio. 
Palabras claves: Comunicación terapéutica; profesional de enfermería; perspectiva; persona hospitalizada.

\begin{abstract}
:
Objective: Determine the level of therapeutic communication professional nursing from the perspective of people hospitalized in the Internal Medicine Service of the General Hospital Jaén, Peru.

Material and method: Research quantitative, descriptive level; an observational, prospective and cross-sectional study, univariate statistical analysis. The sample was composed of 122 people hospitalized, It was used as an instrument of the "therapeutic communication Questionnaire" developed by Lourdes Guevara Chuquillanqui, which was validated by expert judgement as VC $=2.2>\mathrm{VT}=1.6449$ and a reliability of 0.92 .
\end{abstract}

Results: $100 \%$ (122) of persons hospitalized; $71.3 \%$ (87) perceived a therapeutic communication of low level and the $28.7 \%$ (35) medium level. According to the dimensions: In empathy 86 (70.5\%) perceived a therapeutic communication of low level, the 27\% (33) Medium level and 2.5\% (3) high level, in the respect dimension $67(54.9 \%)$ perceived a therapeutic communication a low level, the $42.6 \%(52)$ a medium level and 2.5\% (3) high level, in the dimension receptive listening 67 (54.9\%) perceived a therapeutic communication of low level and the $45.1 \%$ (55) medium level, in the emotions of the patient $70(57.4 \%)$ perceived a therapeutic communication of low level, the $34.4 \%$ (42) Medium level and $8.2 \%$ (10) high level, in the dimension accompanying upon their reflection the $68 \%$

(83) earned a therapeutic communication of low level, the $68 \%$ (83) low level, $25.4 \%$ (31) Medium level and $6.6 \%(8)$ high level.

Conclusions: In conclusion the perception of therapeutic communication of persons hospitalized was of low to medium level.

Keywords: Therapeutic communication; professional nursing; perspective; person hospitalized.

\title{
INTRODUCCIÓN
}

En el ámbito de la oferta sanitaria, muchos problemas se inician cuando la comunicación es deficiente y notoria. Uno de ellos es la inadecuada comunicación con los profesionales de salud, que es la causa principal de insatisfacción de las personas hospitalizadas. Existen estudios que demuestran que entre un 30 a $50 \%$ de estas no muestran adhesión al tratamiento a consecuencia de una deficiente comunicación con el personal ${ }^{(1)}$.

Aunque la comunicación ya es reconocida como esencial, estudios recientes indican que la relación interpersonal entre personas hospitalizadas, profesionales de enfermería y demás profesionales de salud aún no es prioridad en la práctica; en la cual se observa dificultades de comunicación entre los profesionales y las personas hospitalizadas, lo que puede tener fuerte influencia en la satisfacción, la adhesión al tratamiento $\mathrm{y}$, consecuentemente, en los resultados de salud, sugiriendo la necesidad de un cambio teórico-práctico y humanizado de los profesionales de la salud ${ }^{(2,3)}$.

Según estudios realizados en Chile, las personas hospitalizadas realizan su percepción de mala calidad de cuidado expresada como la "falta de atención" o el "trato frío", refieren que es constante en pacientes hospitalizados del servicio de cirugía $(42,3 \%)$ que en medicina $(37,5 \%)^{(4)}$.

El cuidado humanizado del profesional de enfermería tiene una relación muy ligada con la comunicación terapéutica, la cual debe ser una cualidad importante de dicho profesional debido que sirve de gran ayuda al paciente para su recuperación y adaptación, para que el mensaje que se transmite al paciente sea entendido de forma correcta, se debe establecer una relación de ayuda para que el mensaje sea 
entendido en su totalidad (5); en la actualidad muchos de los profesionales de enfermería se han vueltos rutinarios, centrándose solo en la administración de medicamentos, llenados de historias clínicas, olvidándose de brindar una comunicación terapéutica efectiva, dejando de lado su bienestar y la del paciente.

Con el presente trabajo se pretende concientizar a los profesionales de enfermería para así generar cambios en el futuro interviniendo de manera positiva en la relación profesional de enfermería-persona hospitalizada, que es la comunicación terapéutica en la labor de enfermería.

Ante estos argumentos se formuló la siguiente interrogante para realizar esta investigación: ¿Cómo es la comunicación terapéutica del profesional de enfermería, desde la perspectiva de las personas hospitalizadas en el servicio de medicina del Hospital General Jaén, Perú?, considerando que la opinión y experiencia de las personas hospitalizadas es un indicador importante de la comunicación terapéutica.

\section{MATERIAL Y MÉTODOS}

\section{Tipo y diseño de investigación}

Investigación cuantitativa; descriptiva; de tipo: observacional, prospectivo, transversal, según el número de variables de interés y el análisis estadístico univariado ${ }^{6}$.

El diseño de investigación fue descriptivo, (6) cuyo diagrama fue: M: Personas hospitalizadas en el servicio de medicina, O: Perspectiva sobre la comunicación terapéutica del profesional de enfermería.

\section{Población y muestra}

Universo / Población: 177 personas hospitalizadas en el servicio de medicina del Hospital General Jaén en el mes de abril, durante los años 2013 - 2016. (Fuente: Filtro de datos estadísticos en Excel de ingresos y egresos del HGJ).

Muestra: 122 personas hospitalizadas aplicando fórmula para la selección de muestra.

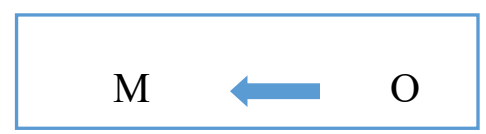

Fórmula para hallar la muestra: $n^{\circ}=\frac{Z^{2} p q N}{e^{2}(N-1)+Z^{2}} \quad p q$

Donde:

$$
n^{\circ}=\frac{1,96^{2} \times 0,5 \times 0,5 \times 1,77}{0,05^{2}(1,77-1)+1,96^{2} \times 0,5 \times 0,5}
$$

$Z=1,96$

$\mathrm{p}=0,5$

$q=0,05$

$\mathrm{n}^{\circ}=$ Muestra

$n^{\circ}=\frac{1,96^{2} \times 0,5 \times 0,5 \times 1,77}{0,05^{2}(1,77-1)+1,96^{2} \times 0,5 \times 0,5}$

$\mathrm{N}=$ Población

$$
\begin{aligned}
& n^{\circ}=\frac{169,92}{0,44+0,96} \\
& n^{\circ}=121,3
\end{aligned}
$$$$
n^{\circ}=121
$$ 
Muestreo: muestreo por conveniencia, usando los criterios de inclusión y exclusión.

\section{En los criterios de inclusión}

- Las personas hospitalizadas en el servicio de medicina que quieran participar a llenar el cuestionario.

- Mínimo cuatro días de hospitalización.

- Edad comprendida entre los 18 a 59 años.

\section{En los criterios de exclusión}

- Personas que no saben escribir o leer.

- Aquellas que no se encuentren en facultad de responderlo (personas en coma, ACV, en estado terminal, entre otros).

\section{Métodos, técnicas e instrumento de recolección de datos}

Método de la encuesta y la técnica del cuestionario (7)

\section{Instrumento de recolección de datos}

Instrumento conformado por 25 ítems compuesto por cinco dimensiones de la comunicación terapéutica enfermero-persona hospitalizada. Se utilizó una escala ordinal, con puntuación para cada ítem de

Nunca . 1

A veces: 2

Siempre 3

El rango real del instrumento fue:

- Puntaje máximo: 75 puntos

- Puntaje mínimo: 25 puntos

\section{Validez y confiabilidad del instrumento}

Se utilizó la encuesta validada por la Lic. Lourdes Guevara Chuquillanqui (8) modificada tipo escala de Likert, que consta de presentación, instrucciones, datos generales y el contenido que cuenta con 25 ítems y 3 alternativas de respuesta: siempre, a veces, nunca; el cual fue sometido a validez de contenido y constructo mediante el juicio de ocho expertos profesionales del área, procesada en la tabla de concordancia y la prueba binomial, validado con un puntaje de 2.2. Posterior a ello, se realizó la prueba piloto a fin de determinar la confiabilidad estadística mediante la aplicación del coeficiente de alfa de Cronbach obteniendo una confiabilidad global de 0.92 .

\section{Análisis de datos}

Para el análisis de datos se utilizó la estadística descriptiva de frecuencias aplicando pruebas de tendencia central como la media, moda y la mediana. Para el 
procesamiento de datos obtenidos se analizó estadísticamente con ayuda de SPSS y la hoja de cálculo Excel $2016^{(9)}$

\section{RESULTADOS}

Tabla 1: Porcentaje de comunicación terapéutica del enfermero, desde la perspectiva de los hospitalizados en el Hospital General Jaén, Perú.

\begin{tabular}{|c|c|c|}
\hline Comunicación terapéutica & $\mathbf{f i}$ & $\%$ \\
\hline Bajo & 87 & 71.3 \\
\hline Medio & 35 & 28.7 \\
\hline Alto & 0 & 0 \\
\hline TOTAL & $\mathbf{1 2 2}$ & $\mathbf{1 0 0}$ \\
\hline
\end{tabular}

Moda $=1$, Mediana $=1$, Media $=1,3$

Fuente: Instrumento de evaluación de comunicación terapéutica.

Figura 1: Porcentaje de comunicación terapéutica del enfermero, desde la perspectiva de los Hospitalizados en el Hospital General Jaén, Perú. El nivel que domino.

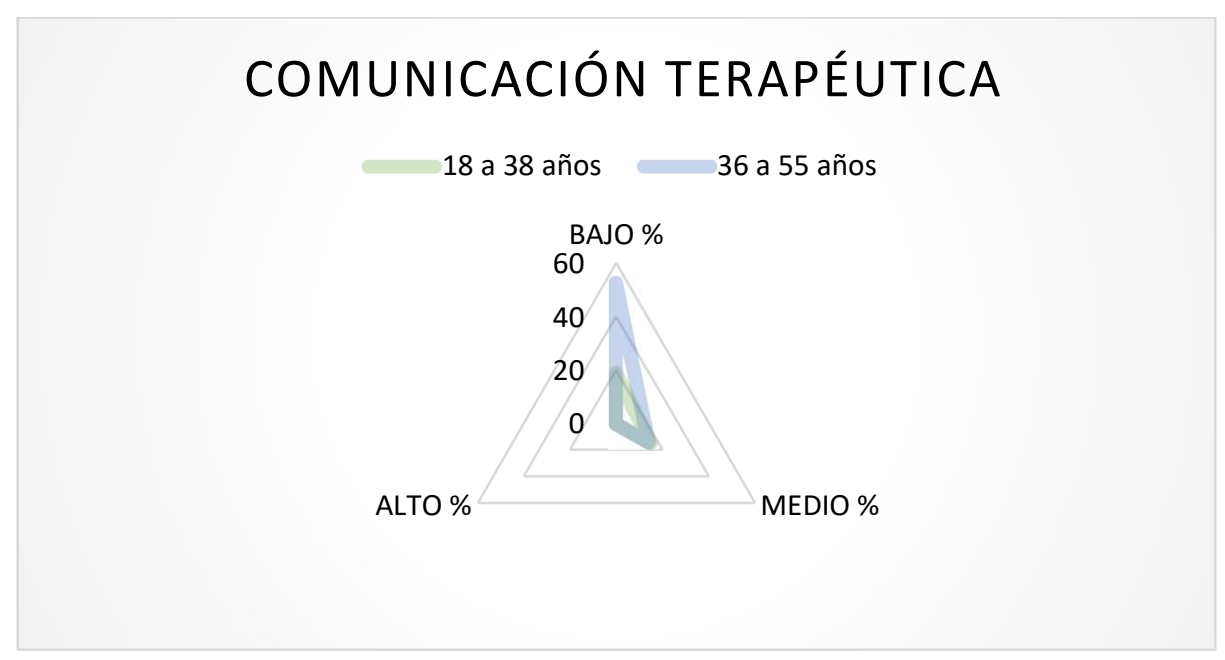

Fuente: Elaboración propia.

En la tabla 1 y figura 1 con respecto al porcentaje de comunicación terapéutica se puede observar que del $100 \%$ (122) de las personas hospitalizadas, el $71.3 \%$ (87) percibió una comunicación terapéutica de nivel bajo, esto quiere decir que el enfermero muestra poco interés, aceptación, entendimiento, brinda mal trato, poca compañía y tiempo, mientras que el $28.7 \%$ (35) presenta un nivel medio. 
Tabla 2: Dimensiones de la comunicación terapéutica del enfermero, desde la perspectiva de los hospitalizados en el Hospital General Jaén, Perú.

\begin{tabular}{|c|c|c|c|c|c|c|c|c|c|c|}
\hline \multirow{2}{*}{$\begin{array}{c}\text { COMUNICACIÓN } \\
\text { TERAPÉUTICA }\end{array}$} & \multicolumn{2}{|c|}{ Empatía } & \multicolumn{2}{|c|}{ Respeto } & \multicolumn{2}{|c|}{$\begin{array}{c}\text { Esch. } \\
\text { Recep. }\end{array}$} & \multicolumn{2}{|c|}{$\begin{array}{c}\text { Emoc. } \\
\text { Pcte. }\end{array}$} & \multicolumn{2}{|c|}{ Acomp. } \\
\cline { 3 - 13 } & $\mathbf{f i}$ & $\%$ & $\mathbf{f i}$ & $\%$ & $\mathbf{f i}$ & $\%$ & $\mathbf{f i}$ & $\%$ & $\mathbf{f i}$ & $\%$ \\
\hline Bajo & 86 & 70.5 & 67 & 54.9 & 67 & 54.9 & 70 & 57.4 & 83 & 68 \\
\hline Medio & 33 & 27 & 52 & 42.6 & 55 & 45.1 & 42 & 34.4 & 31 & 25.4 \\
\hline Alto & 3 & 2.5 & 3 & 2.5 & 0 & 0 & 10 & 8.2 & 8 & 6.6 \\
\hline TOTAL & $\mathbf{1 2 2}$ & $\mathbf{1 0 0}$ & $\mathbf{1 2 2}$ & $\mathbf{1 0 0}$ & $\mathbf{1 2 2}$ & $\mathbf{1 0 0}$ & $\mathbf{1 2 2}$ & $\mathbf{1 0 0}$ & $\mathbf{1 2 2}$ & $\mathbf{1 0 0}$ \\
\hline
\end{tabular}

Esch. Recep.: Escucha receptiva, Emoc. Pcte: Emoción del paciente, Acomp. Reflex.: Acompañamiento en su reflexión.

Figura 2: Dimensiones de la comunicación terapéutica del enfermero desde la perspectiva de los hospitalizados en el Hospital General Jaén, Perú

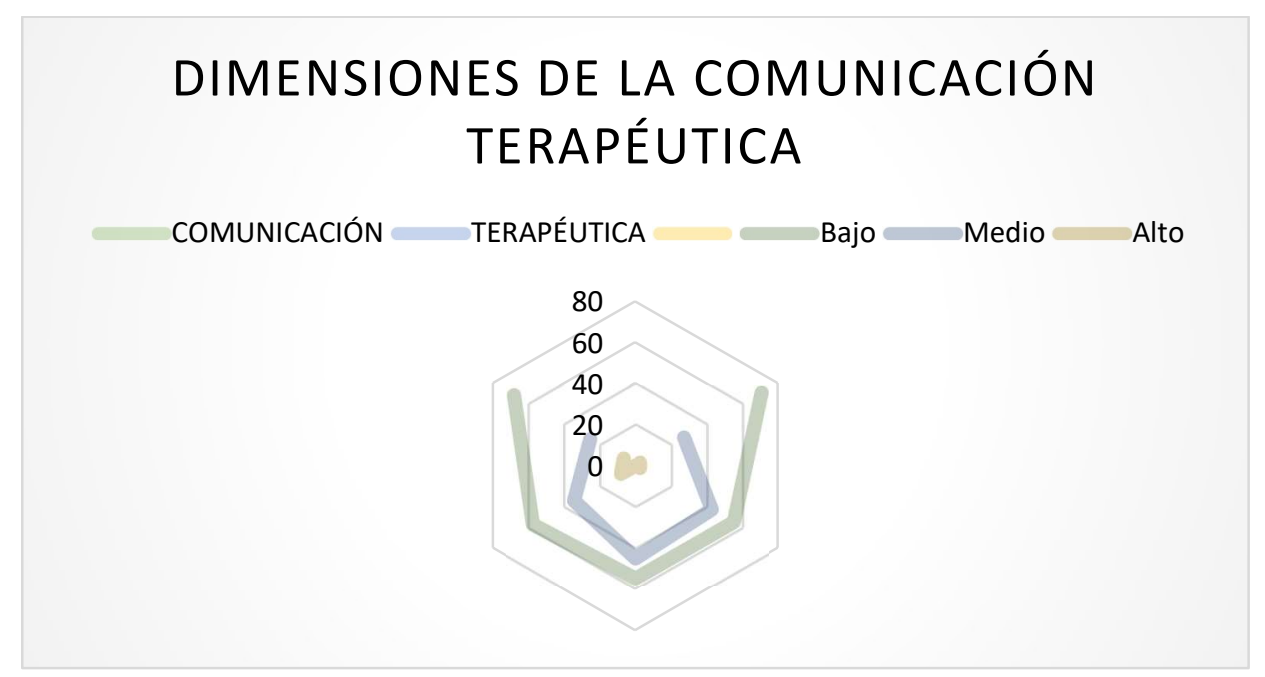

Fuente: Elaboración propia

Con respecto a las dimensiones de comunicación terapéutica, en la tabla 2 y figura 2 se puede observar que del 100\% (122) de las personas hospitalizadas, los valores dominantes que percibieron fue una comunicación terapéutica de nivel bajo para las dimensiones empatía respeto, escucha receptiva y emoción del paciente y el acompañamiento en su reflexión y, en segundo lugar, el nivel medio para todas las dimensiones. 
Tabla 3: Relación entre la edad de las personas hospitalizadas y su percepción de la comunicación terapéutica del enfermero, Hospital General Jaén, Perú

\begin{tabular}{|c|c|c|c|c|c|c|c|}
\hline \multicolumn{8}{|c|}{$\begin{array}{l}\text { RELACIÓN EDAD PERSONAS HOSPITALIZADAS y PERCEPCIÓN } \\
\text { CTE }\end{array}$} \\
\hline \multirow{2}{*}{ EDAD } & \multicolumn{2}{|c|}{ BAJO } & \multicolumn{2}{|c|}{ MEDIO } & \multicolumn{2}{|c|}{ ALTO } & \multirow[t]{2}{*}{ TOTAL } \\
\hline & fi & $\%$ & fi & $\%$ & fi & $\%$ & \\
\hline 18 a 38 años & 23 & 18.9 & 18 & 14.8 & U & 0 & 41 \\
\hline 36 a 55 años & 64 & 52.5 & 17 & 13.9 & 0 & 0 & 81 \\
\hline TOTAL & 87 & 71.3 & 35 & 28.7 & 0 & 0 & 122 \\
\hline
\end{tabular}

Fuente: Instrumento aplicado $x^{2}=6,987^{a}$

Figura 3: Relación entre la edad de las personas hospitalizadas y su percepción de la comunicación terapéutica del enfermero en el Hospital General Jaén, Perú

\section{RELACIÓN EDAD PERSONAS HOSPITALIZADAS Y PCTE}

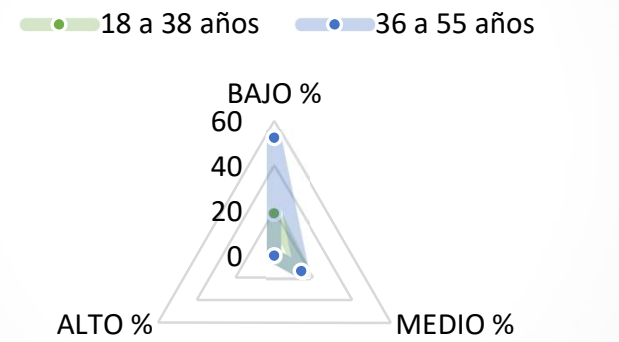

Los pacientes hospitalizados entre 36 a 55 años consideran un nivel bajo de comunicación, con tan solo un $13.4 \%$ de nivel medio, según lo refleja la tabla 03.

Tabla 4: Relación entre la procedencia de las personas hospitalizadas y su percepción de la comunicación terapéutica del enfermero en el Hospital General Jaén, Perú

\begin{tabular}{ccccccccc}
\hline \multicolumn{1}{c}{ RELACIÓN PROCEDENCIA DEL HOPITALIZADO Y PCTE } \\
\cline { 1 - 7 } PROCEDENCIA & \multicolumn{3}{c}{ BAJO } & \multicolumn{2}{c|}{ MEDIO } & \multicolumn{2}{c|}{ ALTO } & \multirow{2}{*}{ TOTAL } \\
\cline { 2 - 6 } & $\mathrm{fi}$ & $\%$ & $\mathrm{fi}$ & $\%$ & $\mathrm{fi}$ & $\%$ & \\
\hline Jaén & 46 & 37.7 & 4 & 3.3 & 0 & 0 & 50 \\
\hline San Felipe & 8 & 6.6 & 11 & 9 & 0 & 0 & 19 \\
\hline Bellavista & 19 & 15.6 & 4 & 3.3 & 0 & 0 & 23 \\
\hline $\begin{array}{c}\text { San José de } \\
\text { Alto }\end{array}$ & 11 & 9 & 16 & 13.1 & 0 & 0 & 27 \\
\hline Santa Rosa & 3 & 2.5 & 0 & 0 & 0 & 0 & 3 \\
\hline TOTAL & 87 & 71.3 & 35 & 28.7 & 0 & 0 & 122 \\
\hline
\end{tabular}

Fuente : Instrumento aplicado $x^{2}=33,359$ 
Figura 4: Relación entre la procedencia de las personas hospitalizadas y su percepción de la comunicación terapéutica del enfermero en el Hospital General Jaén, Perú.

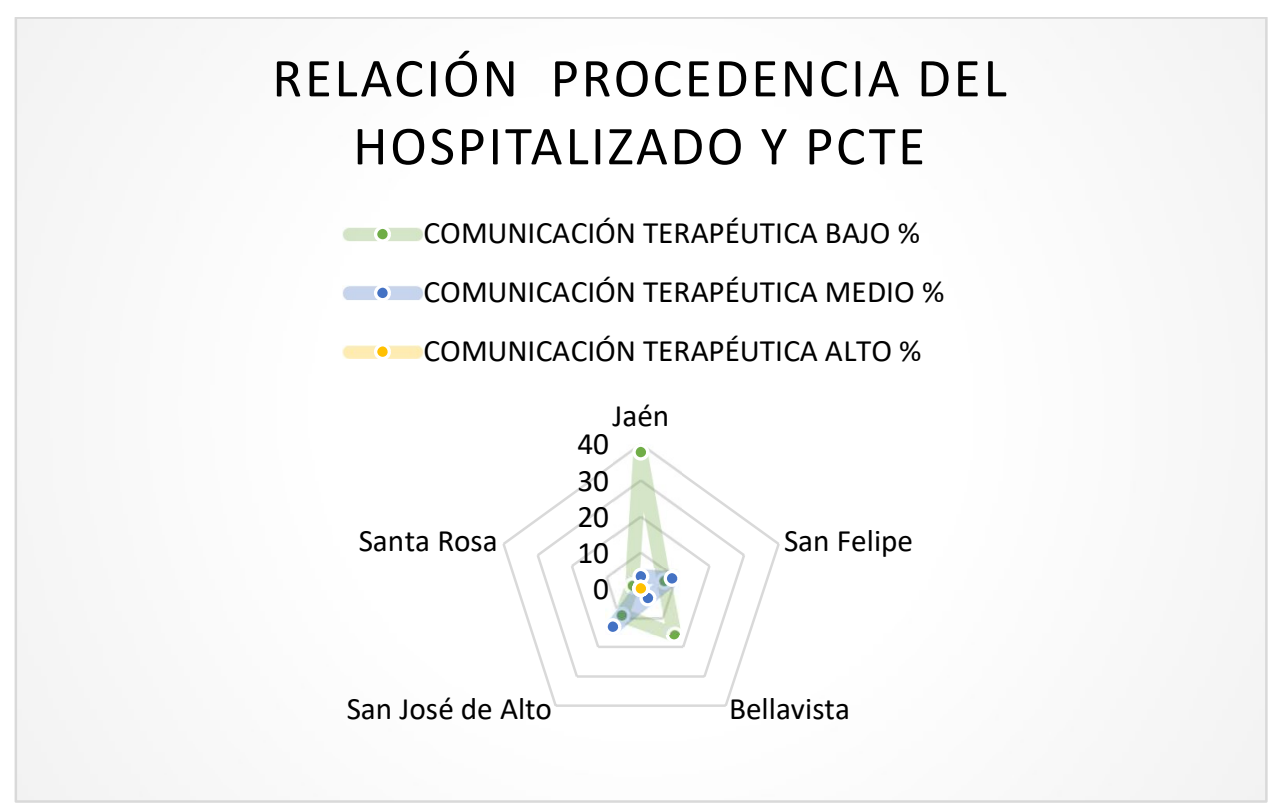

Fuente: Elaboración propia

En cuanto a la procedencia, las personas hospitalizadas de Jaén y Bellavista consideran en su mayoría un nivel bajo, pero San Felipe y San José del Alto consideran un nivel medio según la tabla 4.

Tabla 5: Relación entre la situación laboral de las personas hospitalizadas y su percepción de la comunicación terapéutica del enfermero, Hospital General Jaén, Perú.

\begin{tabular}{|c|c|c|c|c|c|c|c|}
\hline \multicolumn{8}{|c|}{$\begin{array}{c}\text { RELACIÓN ENTRE LA SITUACIÓN LABORAL DE LOS HOPITALIZADOS Y } \\
\text { PERCEPCIÓN CTE }\end{array}$} \\
\hline \multirow{2}{*}{ SITUACIÓN LABORAL } & \multicolumn{2}{|c|}{ BAJO } & \multicolumn{2}{|c|}{ MEDIO } & \multicolumn{2}{|c|}{ ALTO } & \multirow[t]{2}{*}{ TOTAL } \\
\hline & $\mathrm{fi}$ & $\%$ & $\mathrm{fi}$ & $\%$ & $\mathrm{fi}$ & $\%$ & \\
\hline Si trabaja & 76 & 62.3 & 20 & 16.4 & 0 & 0 & 96 \\
\hline No trabaja & 11 & 9 & 15 & 12.3 & 0 & 0 & 26 \\
\hline TOTAL & 87 & 71.3 & 35 & 28.7 & 0 & 0 & 122 \\
\hline
\end{tabular}


Figura 5: Relación entre la situación laboral de los hospitalizados y su percepción de la comunicación terapéutica del enfermero en el Hospital General Jaén, Perú

\section{RELACIÓN ENTRE LA SITUACIÓN LABORAL DE LOS HOSPITALIZADOS Y PCTE}

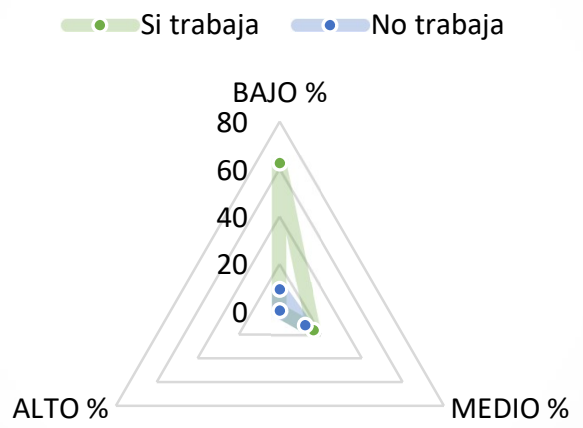

Fuente: Elaboración propia

En cuanto a la situación laboral, dominan las personas que trabajan considerando la existencia de un nivel bajo en comunicación, con un $16.4 \%$ del nivel medio, según lo muestra la tabla 5 .

Tabla 6: Relación entre el estado civil de las personas hospitalizadas y su percepción de la comunicación terapéutica del enfermero, Hospital General Jaén, Perú.

\begin{tabular}{ccccccccc|}
\hline \multicolumn{1}{c}{ RELACIÓN ENTRE EL ESTADO CIVIL DE LOS HOSPITALIZADOS Y PCTE } \\
\hline \multirow{2}{*}{ SITUACIÓN LABORAL } & \multicolumn{2}{c}{ BAJO } & \multicolumn{2}{c|}{ MEDIO } & ALTO & TOTAL \\
\cline { 2 - 9 } & $\mathrm{fi}$ & $\%$ & $\mathrm{fi}$ & $\%$ & $\mathrm{fi}$ & $\%$ & \\
\hline Casado (a) & 87 & 71.3 & 30 & 24.6 & 0 & 0 & 117 \\
\hline Soltero (a) & 0 & 0 & 5 & 4.1 & 0 & 0 & 5 \\
\hline TOTAL & 87 & 71.3 & 35 & 28.7 & 0 & 0 & 122 \\
\hline
\end{tabular}

Fuente: Instrumento aplicado $\quad x^{2}=12,960^{a}$ 
Figura 6: Relación entre el estado civil de los hospitalizados y su percepción de la comunicación terapéutica del enfermero en el Hospital General Jaén, Perú

\section{RELACIÓN ENTRE EL ESTADO CIVIL DEL HOSPITALIZADO Y PERCEPCIÓN CTE}

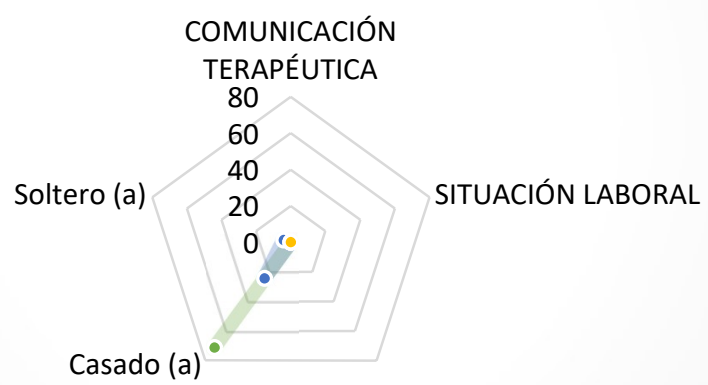

Fuente: Elaboración propia

Todas las personas solteras consideran un nivel medio de comunicación terapéutica por parte del enfermero, pero representan una mínima cantidad con respecto a los casados, quienes consideran la comunicación en un nivel bajo, según la tabla 6 .

Tabla 7: Relación entre los días de hospitalización de las personas hospitalizadas y su percepción de la comunicación terapéutica del enfermero, Hospital General Jaén, Perú.

\begin{tabular}{|ccccccccc|}
\hline RELACIÓN ENTRE LOS DÍAS DE HOSPITALIZACIÓN Y PERCEPCIÓN CTE \\
\cline { 1 - 7 } DÍAS DE & \multicolumn{2}{c}{ BAJO } & \multicolumn{1}{c|}{ MEDIO } & \multicolumn{2}{c|}{ ALTO } & TOTAL \\
\cline { 2 - 7 } HOSPITALIZACIÓN & $\mathrm{fi}$ & $\%$ & $\mathrm{fi}$ & $\%$ & $\mathrm{fi}$ & $\%$ & \\
\hline 4 DÍAS & 77 & 63.1 & 25 & 20.5 & 0 & 0 & 102 \\
\hline 5 DÍAS & 3 & 2.5 & 10 & 8.2 & 0 & 0 & 13 \\
\hline 6 DÍAS & 7 & 5.7 & 0 & 0 & 0 & 0 & 7 \\
\hline TOTAL & 87 & 71.3 & 35 & 28.7 & 0 & 0 & 122 \\
\hline
\end{tabular}

Fuente: Instrumento aplicado $\quad x^{2}=18,471^{\text {a }}$ 
Figura 7: Relación entre los días de hospitalización de los pacientes y la percepción de la comunicación terapéutica del enfermero en el Hospital General Jaén, Perú.

\section{RELACIÓN ENTRE LOS DÍAS DE HOSPITALIZACIÓN Y PERCEPCIÓN CTE}

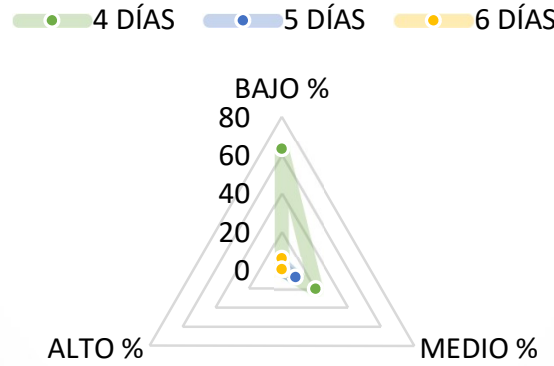

Fuente: Elaboración propia

La relación entre los días de hospitalización de los pacientes y la percepción de la comunicación terapéutica del enfermero estuvo, en su mayor parte, distribuida en los cuatro días de hospitalización, siendo catalogada como baja, lo que refleja la poca empatía de los enfermeros durante los primeros días.

\section{DISCUSIÓN}

Al contrastar con otras investigaciones como la de $\operatorname{Vargas}^{(10)}$, en su estudio Percepción y satisfacción sobre el trato recibido del enfermero en personas cuidadas en el Hospital María Auxiliadora-Rodríguez de Mendoza (Amazonas), se observa que del $100 \%$ de los hospitalizados (65), el $50.8 \%$ (33) tiene una percepción regular del trato recibido por el enfermero; el $49.2 \%$ (32), una percepción positiva sin ninguna evidencia negativa, lo que demuestra que el enfermero se dedicaba al paciente. En el estudio de Huamán (11), titulado Percepción de la persona hospitalizada sobre el cuidado humanizado de enfermería en el servicio de cirugía general. Hospital Regional Virgen de Fátima, Chachapoyas, del $100 \%$ (90) de las personas hospitalizadas, el $74,4 \%$ (67) percibió un cuidado humanizado, seguido de un $15,6 \%$ (14) que lo calificó de excelente y de un $10 \%$ (9) de regular. Cabe resaltar que, a diferencia del Hospital General de Jaén (Perú), estos hospitales -el de Amazonas y Chachapoyas- disponen de un gran número de enfermeros en el cual su labor principal es dedicarse al cuidado de las personas hospitalizadas, dispuestos a satisfacer sus necesidades expuestas por cada una de estas. Sin embargo, la demanda de pacientes, la rutina laboral y el tiempo reducido por cada turno es un limitante para que el profesional de enfermería realice sus funciones adecuadamente. En el área de cirugía esta variable está más avanzada en su deterioro, según estudios realizados en Chile ${ }^{(12)}$.

Por otro lado, Ynga, ${ }^{(13)}$ en su estudio Percepción de la persona hospitalizada sobre su interrelación con el profesional de enfermería. Hospital Regional Virgen de Fátima (Chachapoyas) se encontró que del 100\% (111) de las personas, el 55\% (61) 
establece una relación desfavorable paciente-enfermero, el 45\% (50) muestra una percepción favorable sobre esta interrelación; esto demuestra que, entre más personas encuestadas, los resultados se asemejan a los de esta investigación. Los resultados varían según los días de hospitalización y la cantidad y calidad de profesionales que se encargan del cuidado de las personas y el servicio en el que se encuentren.

Grados ${ }^{(14)}$ encontró que el 100\% (45) de los pacientes calificaron que más del 60\% de la comunicación verbal del enfermero es inadecuada. En la dimensión no verbal, más del $68 \%$ de los pacientes calificaron la comunicación del enfermero como "inexpresiva", de "postura de retirada", mirada "esquiva" y "desatenta". Según este estudio, los enfermeros muestran una debilidad en su comunicación terapéutica, y ante la teoría de las relaciones interpersonales Hildegard E. Peplau, se llega a la misma conclusión debido a que no ejercen una comunicación terapéutica eficaz con las personas hospitalizadas, pues no llegan a reconocer sus dificultades y necesidades, muestran poco interés en la situación del hospitalizado y no usan en un $100 \%$ los servicios. Por último, la persona hospitalizada asume su independencia de manera regular, según resultados de Guevara( ${ }^{(8)}$. Con respecto a la calidad de la interrelación enfermera -paciente, un promedio de $60 \%$ (12) establece una calidad de la interrelación mediana; un promedio de 30\% (6), una calidad de la interrelación como deficiente y un $10 \%(2)$, una calidad de la interrelación óptima.

Durante el proceso de cuidado al hospitalizado se manifiestan factores como el estrés y la ansiedad relacionados con la agresividad del tratamiento, el diagnóstico, la idea de la posible muerte y el deterioro físico-mental posterior al tratamiento(15). En ese proceso, la comunicación terapéutica se presenta como un vínculo entre el paciente y la enfermera, un elemento indispensable en la calidad de las relaciones y en la identificación del proceso salud-enfermedad, puesto que determina una práctica humanizada. Para que la comunicación terapéutica sea efectiva, la enfermera necesita comprender al ser humano como un ser activo y concebir el cuidado al paciente como su máxima finalidad en las más variadas situaciones de la vida (Stefanelly, M.). También se debe considerar que el paciente hospitalizado necesita ser escuchado las veces que sea necesario para sentirse bien y sin preocupaciones, ya que el hecho de estar en un hospital sin poder salir ni realizar sus actividades con normalidad, ya es de por sí una experiencia traumática. ${ }^{(16)}$ Por ello, se realizó esta investigación para visualizar el nivel en el que se encuentra la comunicación terapéutica entre el profesional de enfermería y la persona hospitalizada, y así sugerir algunas recomendaciones para mejorar la calidad de cuidado humanizado.

Desde la perspectiva de las personas hospitalizadas en el Hospital General Jaén, (tabla y figura 2), se puede observar que del $100 \%$ (122) de las personas hospitalizadas; en la dimensión empatía el $70.5 \%$ (86) percibió una comunicación terapéutica de nivel bajo, el $27 \%$ (33) nivel medio y $2.5 \%$ (3) nivel alto, donde el enfermero demuestra poco interés, entendimiento, confianza, generando poca adaptación que ayude a su mejoría. En la dimensión referente al respeto, el $54.9 \%$ (67) percibió la comunicación terapéutica en un nivel bajo; el 42.6\% (52) nivel medio y el $2.5 \%$ (3) nivel alto, donde el enfermero demuestra poco trato y respeto. En la dimensión escucha receptiva el 54.9\% (67) percibió una comunicación terapéutica de nivel bajo y el $45.1 \%$ (55) nivel medio, donde el enfermero no demuestra escucha, brindando poco alivio, dedicándole poco tiempo. En la dimensión emociones del paciente, el $57.4 \%$ (70) percibió una comunicación terapéutica de nivel bajo, el 34.4\% 
(42) nivel medio y el $8.2 \%$ (10) nivel alto, donde el enfermero demuestra intentar cambiar sus emociones. En la dimensión acompañamiento en su reflexión el $68 \%$ (83) percibió una comunicación terapéutica de nivel bajo, el $25.4 \%$ (31) un nivel medio y $6.6 \%$ (8) nivel alto, donde el enfermero brinda poco trato, no favoreciendo que las personas hospitalizadas puedan observarse a sí mismas.

El cuidado de enfermería se ha globalizado en la administración de medicamentos y el llenado de documentos, dejando de lado la comunicación terapéutica del profesional de enfermería, resultados reflejados en la presente investigación, ya antes expuestos. En la encuesta realizada a las personas hospitalizadas, más del $50 \%$ menciona que la falta de tiempo es la mayor debilidad que tiene el profesional de enfermería en la comunicación terapéutica, la falta de estos en el servicio de medicina y más cuando hay carga laboral, refieren y se observa que la demanda de pacientes es constante y el profesional de enfermería no se abastece para iniciar una interrelación con la persona hospitalizada.

En el estudio de Ramos $^{(17)}$ la percepción del paciente en la dimensión orientación, el $55 \%$ (11) tienen una percepción medianamente favorable; $30 \%$ (6), desfavorable y $15 \%$, (3) favorable. En la dimensión identificación, el 45\% (9) expresa una percepción medianamente favorable, $40 \%$ (8) desfavorable, y 15\% (3) favorable. En la dimensión explotación, $40 \%$ (8) favorable, $30 \%$ (6) medianamente favorable y $30 \%$ (6) desfavorable; en la dimensión resolución, $40 \%$ (8) tuvieron una percepción medianamente favorable, 35\% (7) favorable, $25 \%$ (5) desfavorable. Lo cual, si bien es cierto, no concuerda con los resultados obtenidos en esta investigación pues no maneja las mismas dimensiones, aunque parecidas, ya que se utilizó el mismo instrumento de recolección de datos. Se puede observar, entonces, que hay porcentajes altos en la percepción desfavorable, inferiores a los de percepción favorable, pero se advierte que esta brecha no es tan alta ya que la mayoría de pacientes tiene una percepción de "medianamente favorable" a "desfavorable"; lo cual quiere decir que el profesional de enfermería muestra poco interés, aceptación, entendimiento, brinda un mal trato, poca compañía y tiempo a la persona hospitalizada, interpretación que concuerda con esta investigación.

Comparando con Cohelo(2) en su investigación sobre las técnicas del grupo de expresión, muestra que existe una escucha reflexiva en el servicio de la enfermera y del nutricionista; hecho que no concuerda con esta investigación, pues en la dimensión de escucha receptiva se obtuvo un nivel bajo.

Por otro lado, Rivadeneira y Salazar(18) concluyen que la escucha activa consiste en concentrarse exclusivamente en lo que el paciente está diciendo. Si el enfermero escucha al paciente de forma activa, permite que este se centre en sus ideas preconcebidas, así podrá captar el mensaje desde la perspectiva del paciente, no desde la suya propia. Para ello, debe hacer las preguntas adecuadas, someter sus prejuicios e ideas preconcebidas a crítica cuando sea necesario, tener empatía y mostrarla al paciente. Según los resultados obtenidos, la empatía y la escucha receptiva se ubican en un nivel bajo, lo que demuestra poco interés, entendimiento, confianza, generando poca adaptación que ayude a la mejoría del paciente donde no demuestra escuchar, brindando poco alivio, dedicándole poco tiempo, generando un resentimiento en la persona hospitalizada y un mayor barrera para la comunicación terapéutica. 
De igual manera, la comunicación terapéutica entre enfermera-paciente es fundamental para que el cuidado sea más humano. A través de la comunicación, la enfermera(o) puede reconocer al paciente y de algún modo comprenderlo, identificar sus necesidades más sentidas, y convertirse en un transmisor de lo que requiere, lo cual muchas veces no es fácil de expresar a otras personas del equipo de atención ${ }^{(19)}$. Con la apropiación de esta habilidad, la enfermera(o) es capaz de orientar sus intervenciones con un sentido más profundo, llevando su actuar a la integralidad que es implícita del cuidado. En este sentido, el cuidado implica tener cercanía con el paciente por medio de expresiones verbales y no verbales, que ayudan a crear un vínculo que favorece la colaboración del paciente en el proceso de atención en la búsqueda de su recuperación. Según afirman Fernández y Machado "la comunicación terapéutica promueve confort y alivio al dolor, no solo por cuestiones éticas y humanitarias, sino también debido a la comprobación de su interferencia en el estado físico, social y mental del paciente" (20)

\section{CONCLUSIONES}

Desde la perspectiva del hospitalizado del Hospital General Jaén, Perú, el profesional de enfermería muestra poco interés, aceptación, entendimiento y brinda mal trato, poca compañía y falta de dedicación y tiempo.

El nivel de comunicación terapéutica del enfermero en la dimensión empatía, más de la mitad lo percibe en un nivel bajo; en la dimensión de respeto, lo percibe de un nivel bajo a medio; en la dimensión de escucha receptiva, lo percibe de un nivel bajo a medio; en la dimensión de las emociones del paciente, más de la mitad lo percibe en un nivel bajo; en la dimensión de acompañamiento de su reflexión al paciente, más de la mitad lo percibe en un nivel bajo.

Se recomienda al Hospital General de Jaén, Perú:

- Realizar capacitaciones constantes al profesional de enfermería, con la finalidad de mejorar la comunicación terapéutica.

- Elaborar estrategias para autovisualizarse sobre el cuidado brindado por el profesional de enfermería a las personas hospitalizadas.

Se recomienda a la Facultad de Ciencias de la Salud:

- Implementar en la malla curricular la comunicación terapéutica, para todas sus escuelas.

Se recomienda a la Escuela Profesional de Enfermería:

- Evaluar el trato del estudiante a las personas hospitalizadas en las prácticas pre profesionales.

\section{REFERENCIAS}

1. Vidal R, Adamuz J, Feliu P. Relación terapéutica: el pilar de la profesión enfermera. Enfermería Global. SclELO. 2009. Disponible en: http://scielo.isciii.es/scielo.php?pid=S1695- 61412009000300021\&script=sci_arttext. 
2. Coelho M, Zanetti M, Campos E, De Souza C, Moura M, Parente A. La comunicación terapéutica entre profesionales y pacientes en la atención en diabetes mellitus. Rev. Latino-Am. Enfermagem. 2012. 20(4). Disponible en: http://www.scielo.br/pdf/rlae/v20n4/es_08.pdf.

3. Camarena ER, Hernández FT, Dōci GF. La comunicación humana y su relación con la queja médica. Revista conamed. 2016; 16(3):141-147.

4. Medina G. Relación de percepción del paciente y calidad de la interacción según la teoría de peplau: Hospital dos de mayo-2006. Tesis de maestría. Universidad Nacional del Callao. Perú; 2006.

5. Ramírez $P$, Müggenburg $C$. Relaciones personales entre la enfermera y el paciente. Enfermería universitaria. 2015; 12(3): 134-143.

6 . Supo J. Metodología de la investigación científica para las ciencias de la salud. Perú: Universitaria. 2016.

7. Pineda E, De Alvarado E. Metodología de la investigación. 3a ed. EEUU: Washington D C.; 2008.

8. Guevara L. Calidad de la interrelación enfermera - paciente según la teoría de Peplau en los servicios de cirugía del Hospital Nacional Dos de Mayo - 2004. Tesis de pregrado. Universidad Nacional Mayor de San Marcos. Perú; 2005.

9. Hernández R, Fernández C y Baptista P. México. Metodología de la investigación. 5ta ed. México: McGraw; 2010.

10. Vargas L. Percepción y satisfacción sobre el trato recibido del enfermero en personas cuidadas en el Hospital María Auxiliadora, Rodríguez de Mendoza, Amazonas. Tesis de pregrado. Universidad Nacional Toribio Rodríguez de Mendoza de Amazonas, Perú; 2010.

11. Huamán C. Percepción de la persona hospitalizada sobre el cuidado humanizado de enfermería en el servicio de general. Hospital Regional Virgen de Fátima, Chachapoyas - 2014. Tesis de pregrado. Universidad Nacional Toribio Rodríguez de Mendoza de Amazonas. Perú; 2014.

12. Medina G. Relación de percepción del paciente y calidad de la interacción según la teoría de Peplau: hospital dos de mayo-2006. Tesis de maestría. Universidad Nacional del Callao. Perú; 2006.

13. Ynga J. Percepción de la persona hospitalizada sobre su interrelación con el profesional de enfermería hospital regional Virgen de Fátima, Chachapoyas. Tesis de pregrado. Universidad Nacional Toribio Rodríguez de Mendoza de Amazonas, Perú; 2010.

14. Grados B. Características de la comunicación enfermero-paciente postquirúrgico mediato: servicio de cirugía, Hospital Nacional Dos de Mayo, 2013. Tesis de pregrado. Universidad Nacional Mayor de San Marcos; 2014.

15. Lefkowits C, Solomon C. Cuidados Paliativos en Ginecología y Obstetricia. Obstet Gynecol, 2016; 128(14):03-20.

16. Waldow R. Cuidado humano: la vulnerabilidad del ser enfermo y su dimensión de trascendencia. Index Enferm (Gran). 2014; 23(4):234-238.

17. Ramos S. Percepción del paciente sobre la calidad de relación interpersonal enfermera - paciente, en el servicio de 4to piso este del Instituto Nacional de Enfermedades Neoplásicas, Lima - 2010. Tesis de especialidad. Universidad Nacional Mayor de San Marcos. Perú; 2010.

18. Rivadeneira E, Salazar A. Comunicación terapéutica de enfermería en el cuidado de pacientes sometidos a cateterismo cardiaco del Hospital Almanzor Aguinaga, 2015. Tesis de pregrado. Universidad Católica Santo Toribio de Mogrovejo. Perú; 2016

19. Alarcón Rincón, Laura. Serrato Triana, Silvia Arabelly. Implementación de una guía de comunicación para los pacientes con ventilación invasiva conscientes en la 
unidad de cuidado intensivo. En: Revista avances en enfermería [En línea] Bogotá. Colombia. 2014. P.97-136. Disponible en: http://www.bdigital.unal.edu.co/43076/1/1076651617.2014.pdf. [Citado 8 de febrero de 2016]

20 Fernández Da Cruz, Silva Leticia. Machado R., Carla. Estrés del paciente en UCI: visión de los pacientes y del equipo de enfermería. En: Revista Enfermería Global [En línea] Brasil. 2013.

$P \quad 5$.

Disponible en: http://revistas.um.es/eglobal/article/view/154061 